\title{
HALL TECHNIQUE VERSUS THE CONVENTIONAL STAINLESS STEEL CROWNS RESTORING CARIOUS PRIMARY MOLAR TEETH: A RANDOMIZED CONTROLLED CLINICAL TRIAL
}

\author{
Dina A.A Sharaf ${ }^{1 *}$ BDs, Karin ML. Dowidar ${ }^{2} P h D$, Laila M. El Habashy ${ }^{3} P h D$,
} Hams Abdelrahman ${ }^{4} P h D$

\section{ABSTRACT}

INTRODUCTION: Dental caries is considered the most common chronic disease affecting children globally, management of carious primary molars has evolved and a shift has occurred from the early conventional approaches where carious tissue was completely removed to the more recent biologic/ minimally invasive approaches that require less caries removal or no caries removal; one of the recent approaches is the Hall technique. Objectives: Compare the clinical and radiographic success of the stainless steel crowns (SSCs) placed by the Hall technique (HT) to the conventional method in restoring carious primary molars across one year. Materials and Methods: Parallel randomized controlled trial, participants were allocated in the Faculty of Dentistry, Alexandria university, Egypt. One hundred and four primary molars in four- to eight -year-old children were randomly divided into two treatment groups. Teeth were assessed at one week, one, six and 12 months for clinical success using standardized criteria showing either success, minor or major failure and at six and 12 months for radiographic evaluation. Success/failure rates were compared using chi square test. McNemar's test was applied in testing changes within each group. Results: No significant differences clinically or radiographically between the two groups at all follow ups periods. The HT group and the conventional groups showed (94.2\%) and (88.5\%) success rates respectively. Failures in both groups were major failures. Conclusions: The Hall technique showed comparable results when compared to that of the conventional SSC restorations for treatment of carious primary molar teeth that were not pulpally involved.

KEYWORDS: Hall technique, stainless steel crowns, primary molar teeth

RUNNING TITLE: Clinical and radiographic success of hall technique crowns

1 Assistant lecturer, Pediatric dentistry department, Faculty of Dentistry, Alexandria University, Egypt

2 Professor, Pediatric dentistry department, Faculty of Dentistry, Alexandria University, Egypt

3 Assistant Professor, Pediatric dentistry department, Faculty of Dentistry, Alexandria University, Egypt

4 Assistant lecturer of Dental Public Health. Department of Pediatric Dentistry and Dental Public Health. Faculty of Dentistry, Alexandria University. Egypt.

\section{*Corresponding author}

E-mail:dr.dinasharaf@gmail.com

\section{INTRODUCTION}

Dental caries is reported to be the most common chronic disease affecting children globally. It is a microbiological disease of the teeth that results in localized dissolution and destruction of the sound calcified tissues. Its management and treatment have drawn the attention of many scholars, researchers and dentists worldwide(1).

Over the years the management of carious primary molars has evolved and a paradigm shift has occurred from the early conventional approaches where carious tissue was completely removed and a restoration was placed to the more recent biologic/ minimally invasive approaches that require less caries removal or no caries removal followed by placement of a suitable restoration(2).
Currently the properties and characteristics of dental biofilm and its relationship with tooth decay have received much attention. Some researchers claim that the biofilm plays a major role in dental caries and has many implications in the development of lesions where biofilms are allowed to mature and remain for prolonged periods of time(3). The management of deep carious lesions, approaching a healthy pulp, presents a significant challenge to the dental practitioner as deep caries may induce severe inflammatory reactions in the pulp and may cause pulpal necrosis. While excavating the deep carious lesions, there are chances that the dentin barrier may be broken and the healing of the pulp is impaired(4). 
Ricketts et al (2013) (1) stated that in order to minimize the risk of exposing or even breaching the pulp, alternative approaches such as the Hall Technique in management of carious lesions are of great importance. The novel approaches for caries management have not only restored teeth in a minimal fashion but also contributed greatly in the cooperation of children with carious molars.

The technique is named after Dr Norna Hall, a general dental practitioner from Scotland who had been placing SSCs since 1988 using this new technique where she cemented a crown over a caries-affected primary molar without local anesthetic (LA), caries removal, nor tooth preparation. Stainless steel crowns used by this technique are being referred to as Hall crowns $(5,6)$. Even though the placement of SSCs by the conventional technique has been considered clinically successful, however many clinicians find the crowns difficult to fit due to lack of patient cooperation, prolonged chair time, and/or the need to administer local anesthesia(7).

In the pilot study conducted on this technique. The technique was considered acceptable to the dentists, patients, and parents involved (8). One of the first studies reported a 23-month follow up of the clinical effectiveness of the technique. Patients with caries in primary molars were randomly allocated to the HT group or to the conventional restoration group. Failures were categorized as major or minor; major failures included irreversible pulpitis, dental abscess needing extraction, restoration loss with tooth unrestorable, or internal resorption on radiograph. Minor failures were lost fillings, recurrent caries or new caries, restoration wear, SSCs lost, or caries progress visible on radiograph. At the follow up recalls, the conventional restorations recorded more major and minor failures than the HT, the HT showed more favorable outcomes for pulp health and restoration longevity than conventional restorations, and the conclusion was that the HT appeared to offer a treatment option for caries-affected primary molar teeth (9).

Ludwig et al in 2014 (10) retrospectively assessed the clinical and radiographic success of SSCs placed to restore cariesaffected primary molars using both the traditional placement technique for SSC and Hall crowns. Restoration failure was defined as crown lost (i.e., SSCs became uncemented) or further treatment required (i.e., further treatment apart from recementation and based on radiographic evidence of pulpal pathology or clinical evidence of recurrent caries).. Results showed that success rates for the HT and conventional SSCs were not significantly different.

Since the Hall technique is a novel approach that is minimally invasive and biologic in nature and only few studies were conducted to evaluate its clinical and radiographic success specially after a one year follow up.

Hence the aim of the present study is to record and assess the success and failure in both Hall technique and conventional SSC restorations in the management of carious lesions in primary molars after one year postoperatively. The null hypothesis of this study is that there is no difference between the clinical and radiographic outcomes of the stainless steel crowns placed by the Hall technique or the conventional method in restoring carious primary molars that are not pulpally involved across one year.

\section{MATERIALS AND METHODS}

This study was a two arm, parallel group, blinded, randomized controlled clinical trial.

It was set up and reported according to the CONSORT statement (11). The study design was approved by the Ethics Committee of the Faculty of Dentistry, Alexandria University under the code IRB NO 00010556-IORG0008839. The study is part of a clinical trial registered in archives of ClinicalTrials.gov with registration number : NCT04367649. This trial was conducted between November 2018 and February 2020 in the Pediatric Dentistry Department, Alexandria University. Patient recruitment was conducted by a resident pediatric dentist at the outpatient clinic, Pediatric Dentistry and Dental Public Health Department, Faculty of Dentistry, Alexandria University, Egypt and completed within four months. Written informed consent was obtained from parents or guardians of the children after explaining the protocol, risks and benefits of the study. One molar tooth from each participant was included in the study; participants included were cooperative children (positive/ definitely positive) according to Frankl's behavior rating scale (12) with an age range of 4 to 8 years old and free of any systemic disease or special health care needs. The primary molar tooth had occlusal or occluso-proximal carious lesions into dentin (International Caries Detection and Assessment System) (ICDAS) codes: 3-5 (13) with absence of any clinical signs or symptoms of irreversible pulpitis or fistula/abscess near the selected tooth clinically and radiographically(14). The teeth included shouldn't show any spontaneous pain or pulp exposure with no pathological or physiological mobility (15).

\section{Sample size estimation:}

Sample size calculation was calculated based on the comparison of failure rates for HT (5\%) versus CR (46\%) (9) Failure rates of $5 \%$ for $\mathrm{HT}$ and $25 \%$ for CR were assumed. The program (http://powerandsamplesize.com/calculators/compare-

2Proportions/2-Sample-Equality) was used for sample size calculation (16): 2-tail test, $\alpha=0.05$ "three" for and $\beta=0.20$. This resulted in a sample size of 47 children per group with a total sample size $=94$ to be increased by $10 \%$ to make up for loss to follow up with a required sample size of 104 children.

\section{Participants randomization and allocation:}

The participants complying with the inclusion criteria were randomly assigned into one of the two arms (conventional restorative technique and HT) with equal allocation ratio 1:1. Block randomization was applied with block size of 4 (17) using a computer generated (18) list of random numbers. Allocation was performed by a trial independent individual. Each child included in the study was given a serial number that was used in the allocation. These numbers were written on identical sheets of paper with the group to which each child was allocated. The papers were placed in opaque envelopes carrying the respective names of the children. A trial independent personnel was assigned the role of keeping the envelopes and unfolding them only at the time of treatment (19). The participants prior to treatment and statistician were blinded to the intervention group. 
After data analysis was completed, the randomization code was broken to reveal the allocation group (20).

The Participants were randomly divided into two groups according to the technique used; Conventional restorative treatment $(\mathrm{N}=52)$ and Hall technique ( $\mathrm{N}=52)$ as shown in figure (1)

One trained and calibrated examiner carried out the baseline examination and follow-up evaluations. The operator underwent a training period in the treatment modalities (21). Ten \% of the sample, were assessed clinically and radiographically by the same examiner to investigate the intra-examiner reliability. Kappa Values ranged from 0.78 to 0.83 indicating substantial agreement.

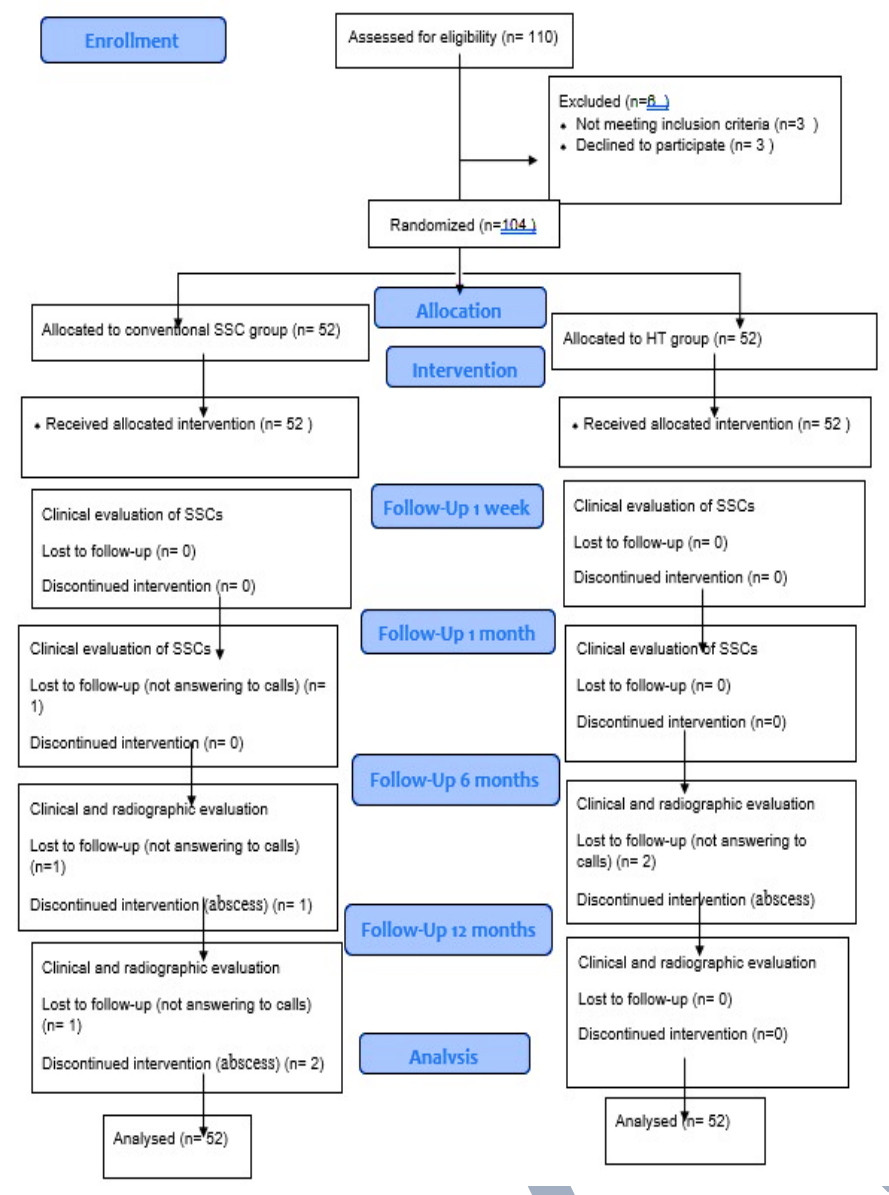

Figure (1) : Flowchart of the treatment groups

In the conventional restorations $(22,23)$ group local anaesthesia (Mepecaine L 1.8ml, Mepevicaine HCl 2\% Levonordefrin 1:20000) and rubber dam isolation was administered, complete caries removal was carried out with a high-speed hand piece, and an excavator to clear carious dentin from the pulpal wall, proximal surfaces were reduced using number 69L bur at high speed, the cusps and the occlusal portion of tooth were then be reduced using a 69L bur revolving at high speed and approximately $1 \mathrm{~mm}$ of clearance with the opposing teeth was achieved. Proper crown size was selected (Stainless Steel Crowns, $3 \mathrm{M}^{\mathrm{TM}}$ ESPETM, St. Paul, MN, USA) and cemented using GIC Fuji IX capsules (GC Fuji IX GP,GC America).
In the Hall Technique group (5) at the first appointment, the calibrated and trained operator assessed the tooth shape, contact points/areas and the occlusion to determine size of the crown. Some cases required orthodontic separators to create space for fitting of the crown. A second appointment was scheduled for the patients with separators after three days to remove them. The airway was protected by placing a gauze swab square between the tongue and the tooth to be crowned and the correct crown size (Stainless Steel Crowns, $3 \mathrm{M}^{\mathrm{TM}}$ ESPETM, St.Paul, MN, USA) was selected and inserted with a slight feeling of "spring back." till reaching the gingival margin. Cementation was done by glass ionomer cement (GC Fuji IX GP,GC America). After insertion of the crowns, the patients' bite was monitored to ensure that incase of an increased occluso-vertical dimension, this did not cause discomfort to the patient. Both treatment groups were assessed every 3 months to ensure proper oral hygiene measures.

\section{Clinical and radiographic outcomes:}

Teeth in both groups were assessed clinically according to the clinical evaluation criteria described by Innes et al (24) as shown in table (1) at one week then at one, six and 12 months postoperatively.

Teeth were radiographically assessed at 6, 12 months postoperatively. Teeth were considered radiographically successful when they showed no evidence of 1 . Radicular radiolucency, 2. Internal or external root resorption, 3. Periodontal ligament space widening (25). In case of failure radiographically with no signs or symptoms of clinical failure, the tooth was also considered to be failing.

Table (1): clinical evaluation criteria of stainless steel crowns (24)

\begin{tabular}{|l|l|l}
\hline Success & Minor Failures: & Major Failures: \\
\hline
\end{tabular}

- Satisfactory - Secondary caries, $\bullet$ Irreversible pulpitis restoration/crown or new lesions or dental not showing any detected clinically fistula/abscess, signs of major or $\bullet$ Crown presents requiring minor failures perforation $\quad$ pulpotomy or according to $\bullet$ Restoration extraction. Innes et al ${ }^{(9)} \quad$ fracture or wear - $\bullet$ Loss of - No clinical signs intervention restoration/crown. or symptoms of required - Tooth not capable pulp pathology. - Loss of restoration of being re-

- tooth that can be restored. re-restored

- Crown loss - tooth able to be retreated

- Reversible pulpitis, which could be treated without the need for extraction or pulpotomy

Data were analyzed with Intention to treat analysis (ITT). Success/failure rates were displayed using count and percent and 
compared using chi square test and McNemar's test was applied in testing changes between two time points within each group. $\mathrm{P}$ value was adjusted for all multiple comparisons using Bonferroni correction method. Significance level was set at p value $\leq 0.05$. Data were analyzed using IBM SPSS statistical software (version 23).

\section{RESULTS}

The present study was conducted on a total of 110 participants; three did not meet the inclusion criteria and three refused to particpate in the study resulting in 104 participants (51 girls and 53 boys). The study included one primary molar for each child participating. The age ranged from 4 to 8 years with a mean age of 5.5 and 5.9 in the HT group and SSC group respectively. At the 1 week recall, all participants were availble for evaluation, 52 patients per group. At the 1 month recall, 52 patients in the HT group and 51 patients in the SSC group were available for evaluation. At the 6 months recall, 50 patients in the HT group and 50 patients in the SSC group were availble for evaluation. At the 12 months recall, 50 patients in the HT group and 49 patients in the SSC group were availble for evaluation. The reasons for dropout included no response to calls or lack of parents' cooperation. The mean age of the participants in the Hall technique group was 5.5 years and 5.9 years in the conventional SSC group. The male patients represented $55.8 \%$ in the Hall group and $46.2 \%$ in the conventional SSC group,the female patients represented $44.2 \%$ and $53.8 \%$ in the Hall group and convrntional SSC group respectively. There were 16 (30.8\%) and 30 (57.7\%) second primary molar teeth in the Hall group and conventional SSC group respectively and 36 (69.2\%) first primary molar teeth in the hall group and $22(42.3 \%)$ in the conventional group.

The HT group: The clinical outcome of the present study shows a (100\%) success rate in the Hall technique group at the 1 week and 1 month follow up visits, at six months the success rate decreased to (94.2\%) where 49 restorations were considered satisfactory and three restorations showed failure ; this failure was due to the drop out of two patients from the clinical trial in the follow up visits and one tooth showed clinical major failure in the form of an abscess. At the 12 months follow up visit a (94.2\%) success rate was recorded where no further participants dropped from the clinical trial nor any other restorations showed clinical failure. No statistically significant difference was found neither between the one month and 12 months follow up nor between the six months and 12 months follow up $\mathrm{p}=1.00$ as shown in table (2).

The conventional SSC group: This group on the other hand showed a (100\%) success rate at the 1 week follow up visit, followed by a (98.1\%) success rate at 1 month due to the drop out of one participant in the follow up visits, next at the six months and 12 months follow up visits success rate was recorded at $(94.2 \%)$ and $(88.5 \%)$ respectively where two additional participants were lost to follow up and three restorations showed clinical major failure in the form of an abscess : one at the 6 months follow up visit and two more at the 12 months follow up visit . No statistically significant difference was found neither between the one month and 12 months follow up $\mathrm{p}=0.063$ nor between the six months and 12 months follow up $\mathrm{p}=0.250$.
When comparing both groups at one week, one month, six months and 12 months there was no statistically significant difference ( $p$ value $>0.05$ ) as shown in table (2).

In terms of radiographic success and failure at different time points (six months and 12 months). There was no statistically significant differences between both groups at six and 12 months ( $\mathrm{p}$ value $>0.05$ ) as shown in table (3).

Table 2: Clinical outcome for the study groups at different time

\begin{tabular}{|c|c|c|c|c|}
\hline \multicolumn{2}{|c|}{} & $\begin{array}{c}\text { Hall } \\
\text { points }\end{array}$ & $\begin{array}{c}\text { Conventional } \\
(\mathbf{n = 5 2 )}\end{array}$ & P value \\
\hline \multirow{2}{*}{1 week } & Success & $52(100 \%)$ & $52(100 \%)$ & \multirow{2}{*}{-} \\
\cline { 2 - 4 } & Failure & $0(0 \%)$ & $0(0 \%)$ & \\
\hline \multirow{2}{*}{1 month } & Success & $52(100 \%)$ & $51(98.1 \%)$ & \multirow{2}{*}{0.315} \\
\cline { 2 - 4 } & Failure & $0(0 \%)$ & $1(1.9 \%)$ & \\
\hline \multirow{2}{*}{6 months } & Success & $49(94.2 \%)$ & $49(94.2 \%)$ & \multirow{2}{*}{1.00} \\
\cline { 2 - 4 } & Failure & $3(5.8 \%)$ & $3(5.8 \%)$ & \\
\hline 12 months & Success & $49(94.2 \%)$ & $46(88.5 \%)$ & 0.295 \\
\cline { 2 - 4 } & Failure & $3(5.8 \%)$ & $6(11.5 \%)$ & \\
\hline \multirow{2}{*}{$\begin{array}{c}\text { P value } \\
(1 \text { month }-12 \text { months })\end{array}$} & - & 0.063 & \\
\hline \multicolumn{2}{|c|}{$\begin{array}{c}\text { P value } \\
\text { (6 month -12 months) }\end{array}$} & 1.00 & 0.250 & \\
\hline
\end{tabular}

Table (3) Radiographic outcome for the study groups at different time points

\begin{tabular}{|c|c|c|c|c|}
\hline \multicolumn{2}{|c|}{ 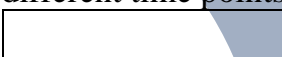 } & $\begin{array}{l}\text { Hall } \\
(n=52)\end{array}$ & $\begin{array}{c}\text { Conventional } \\
(n=52)\end{array}$ & P value \\
\hline \multirow[t]{2}{*}{6 months } & Success & 49 (94.2\%) & 49 (94.2\%) & \multirow[t]{2}{*}{1.00} \\
\hline & Failure & $3(5.8 \%)$ & $3(5.8 \%)$ & \\
\hline \multirow[t]{2}{*}{12 months } & Success & 49 (94.2\%) & 46 (88.5\%) & \multirow[t]{2}{*}{0.295} \\
\hline & Failure & $3(5.8 \%)$ & $6(11.5 \%)$ & \\
\hline \multicolumn{2}{|c|}{ P value } & 1.00 & 0.250 & \\
\hline
\end{tabular}

In the current study, SSC restorations were used as a standard treatment in the control group because it is considered one of the most reliable and durable treatments for carious primary molars (8). The sample size consisted of 104 participants, one carious primary molar per each participant, this was based on the sample sizes of similar previous studies $(2,26)$. The chosen sample age was between 4 to 8 years old to ensure the primary molars are not shedding during the study period. The follow up period and recall appointments were at one week, one month, six months and finally 12 months postoperatively in accordance to most of the studies conducted previously that had a similar followup period $(2,26)$.

In the present study assessment of the success and failure of the HT and the conventional SSC both clinically and radiographically were based upon certain criteria described by Innes et al in 2007 that are considered the gold standard in evaluation of the SSCs placed by both the HT and the conventional method (9).

The null hypothesis in the current study was accepted where no significant difference was found clinically or radiographically between the HT and SSC groups at all the follow up visits. These findings were consistent with the results of several studies 
showing clinical success of the HT versus conventional SSC restorations $(5,10,27)$.

When comparing the clinical outcome of the HT and conventional SSC restorations, HT recorded a $100 \%$ success rate at one week and one month, then decreasing to $94.2 \%$ at six and 12 months. On the other hand the conventional SSC restorations recorded a $100 \%$ success rate at one week and $98 \%$ at one month then $94.2 \%$ and $88.5 \%$ at six months and 12 months respectively. No statistically significant difference was found at all followup visits between both groups, and no significant difference was found at the different time intervals within the same treatment group.

The radiographic outcome of the HT was recorded at $94.2 \%$ success rate at both six and 12 months where 49 teeth were considered successful and only one showed a major failure according to the HT assessment criteria. The coventional SSC restoration also showed a $94.2 \%$ success rate at six months with one tooth showing failure but at 12 months this success rate decreased to $88.5 \%$ where two more teeth showed radiographic failure according to the assessment criteria. There was no significant difference between both groups regarding the radiographic success rate at both followups and no significant difference was found at the different time intervals within the same treatment group.

The HT group showed no minor failures and only one major failure in one tooth represented in an abscess (figure 2), this can be attributed to iatrogenic factors as misdiagnosis where the tooth might have been pulpally involved but showing no signs clinically or radiographically. The success of the SSC placed by the Hall technique can be explained by the accurate diagnosis and the fact that no tooth preparation nor removal of caries is required; this preserves the secondary dentin formed isolating it from the surrounding environment and thus allows no progression of caries. On the other hand the SSC group also showed no minor failures however the major failures (figure 3) in three teeth depicted in an abscess formation; this can be due to pulpal irritation from the close proximity of the high mesial pulp horn to the bur. Teeth that showed clinical and radiographic failure were treated afterwards by either extraction or pulpectomy in both groups.

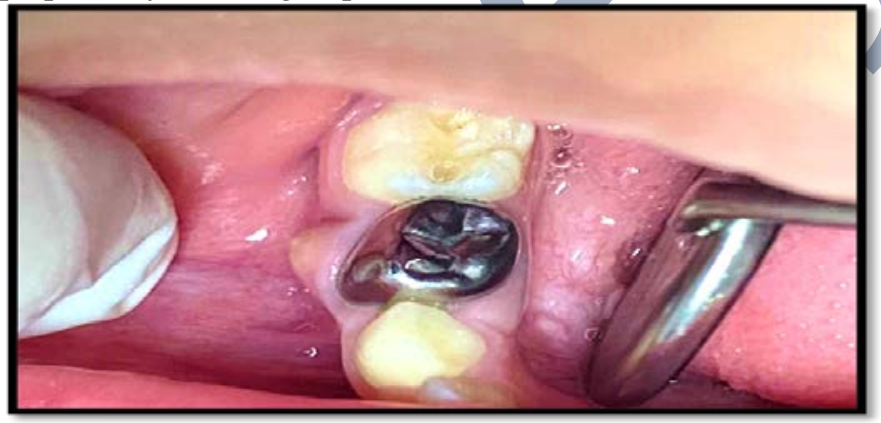

Figure (2): Clinical failure of the stainless steel crown placed by the Hall technique.

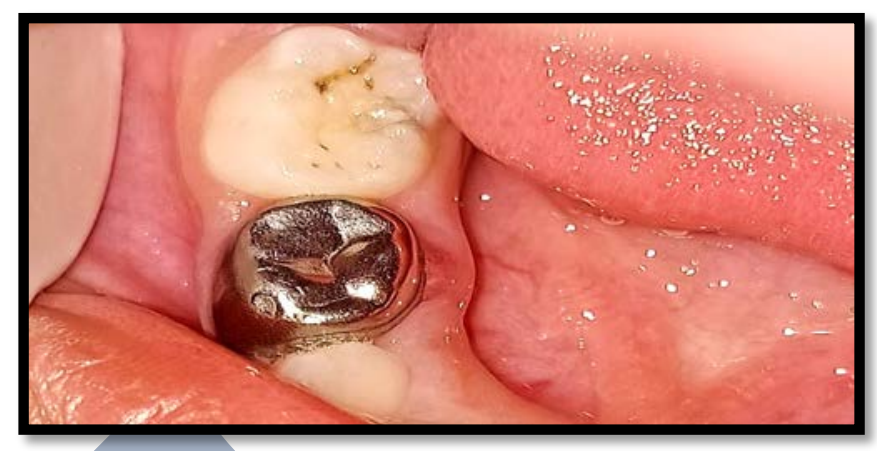

Figure (3): Clinical failure of the stainless steel crown placed by the conventional method (abscess appearing lingually)

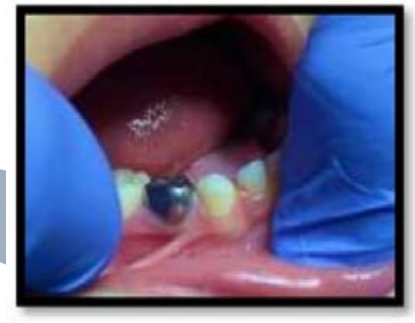

Immediately

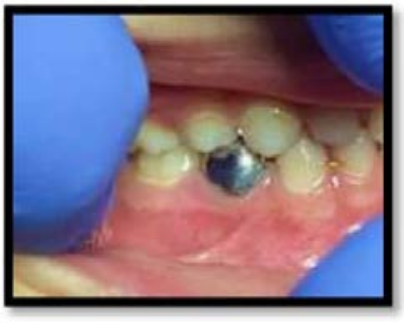

12 months
Figure (4): Clinical performance of the stainless steel crown placed conventionally immediately after insertion and at 12 months postoperatively.
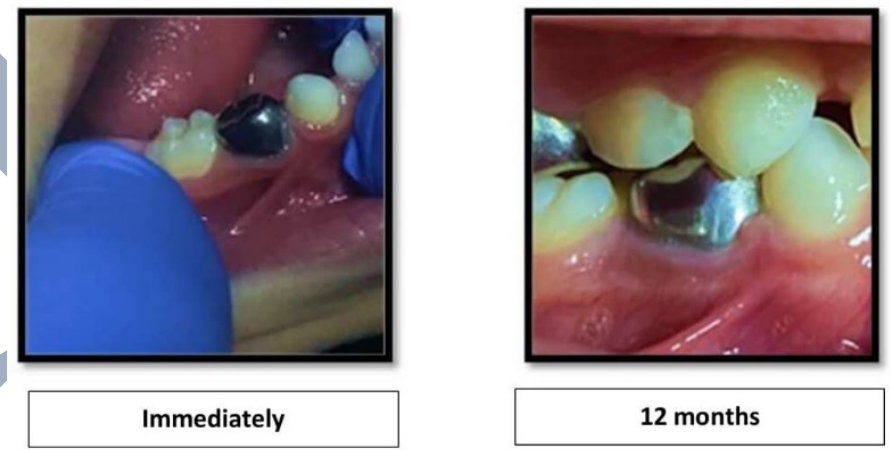

12 months

Figure (5): Clinical performance of the stainless steel crown placed by the Hall technique immediately and at 12 months postoperatively.

This is in agreement with the results of a study by Innes et al 2011(26) who recorded more major failures than minor failures in both groups and concluded that HT has recorded less failures in total than the conventional SSC group; the HT recorded $3 \%$ major failures and $5 \%$ minor failures while the conventional restorations recorded $16.5 \%$ major failures and $42 \%$ minor failures. Major failures ranged between irreversible pulpitis, loss of vitality, abscess, or unrestorable teeth while minor failures ranged between reversible pulpitis, restoration loss/wear/fracture; or secondary caries. 
Results from a study by Ebrahimi et al in 2020 (28) were in accordance with results from the current study where HT showed a $100 \%$ success rate at the 6-month follow up whereas 98.7\% for the conventional SSC restorations due to a major failure (SSC was lost due to lack of retention, and developed an abscess). Whereas at the 12-month follow up of the study $99.1 \%$ success rate was recorded for the HT where failure of the crown was due to crown perforation (minor failure)and $96.1 \%$ for the conventional SSC restoratoration where two more crowns failed because of abscess formation (major failure).

The current results are similar to results of two retrospective studies one by Binladen et al in 2020 (29) where at 12months, 99.1\% of the Hall crowns and $96.1 \%$ of the conventional SSC restorations showed to be successful and observed failure of the Hall crown was the result of a minor failure where the crown was perforated, whereas the conventional SSC restoration failed because of abscess formation. There was no signifcant diference in success/failure at 12 months. And another by Ludwig et al in 2014 (10) where $97 \%$ of the SSCs placed by the Hall technique and $94 \%$ of the SSCs placed traditionally were successful, the two observed failures of Hall technique crowns were the result of abscesses, five conventional SSCs placed failed because of abscess formation at an average while two other crowns showed retention failure.

Based on HT's high success rate and its minimally invasive approach it is suggested that it be a treatment option for carious primary molars that are not pulpally involved.

The Hall Technique has many advantages including high clinical success and could be a suitable treatment option for anxious children with fear of injections or drilling, however the HT requires very accurate and precise diagnosis by conductiong a proper history from the patient and accurate clinical and radiographic examinations to ensure non pulpal involovement. In many cases placement of seperators was mandatory to be removed after three days, this presented a limitation as many of the participants parents required the procedure to be over in the same visit. Therefore proper diagnosis and the placement of seperators were some of the limitations of the present study. It is suggested that, in future studies, the patient and parent satisfaction towards the novel treatment modality to be evaluated in addition to the measurement of the occluso-vertical dimension after insertion of the Hall crowns, in addition to the cost effectivness of both treatment groups and the clinical and radiographic success for longer follow up periods.

\section{CONCLUSION}

Based on this study's results, the following conclusions can be made:

The Hall technique shows acceptable clinical and radiographic results when compared to that of the conventional stainless steel crown restorations for treatment of primary molar teeth with carious lesions that are not pulpally involved.

\section{Funding:}

No institutional funding was provided.

Conflict of interest:

There was no conflict of interest in the following study

\section{REFERENCES}

1. Ricketts D, Lamont T, Innes NP, Kidd E, Clarkson JE. Operative caries management in adults and children. The Cochrane database of systematic reviews. 2013:CD003808.

2. Santamaria RM, Innes NP, Machiulskiene V, Evans DJ, Splieth $\mathrm{CH}$. Caries management strategies for primary molars: 1-yr randomized control trial results. J Dent Res. 2014;93:1062-9.

3. Carvalho T-S, Ribeiro T-R, Bönecker M, Pinheiro E-C-M, Colares V. The atraumatic restorative treatment approach: an "atraumatic" alternative. CEP. 2009;5508:e668-e73.

4. Bjørndal L, Reit C, Bruun G, Markvart M, Kjældgaard M, Näsman P, et al. Treatment of deep caries lesions in adults: randomized clinical trials comparing stepwise vs. direct complete excavation, and direct pulp capping vs. partial pulpotomy. European journal of oral sciences. 2010;118:290-7.

5. Innes NP, Evans DJ, Stewart M. The Hall Technique A minimal intervention, child centred approach to managing the carious primary molar. A users manual. 2015.

6. Innes N, Evans D, Hall N. The Hall Technique for managing carious primary molars. Dent Update. 2009;36:472-4, 7-8.

7. Erdemci ZY, Çehreli SB, Tirali RE. Hall versus conventional stainless steel crown techniques: in vitro investigation of marginal fit and microleakage using three different luting agents. Pediatric dentistry. 2014;36:286-90.

8. Randall RC. Preformed metal crowns for primary and permanent molar teeth: review of the literature. Pediatr Dent. 2002;24(5):489-500.

9. Innes NP, Eyans DJ, Stirrups DR. The Hall Technique; a randomized controlled clinical trial of a novel method of managing carious primary molars in general dental practice: acceptability of the technique and outcomes at 23 months. BMC Oral Health. 2007;7:18.

10. Ludwig KH, Fontana M, Vinson LA, Platt JA, Dean JA. The success of stainless steel crowns placed with the Hall technique: a retrospective study. J Am Dent Assoc. 2014;145:1248-53.

11. Moher D, Hopewell S, Schulz KF, Montori V, Gotzsche PC, Devereaux PJ, et al. CONSORT 2010 explanation and elaboration: updated guidelines for reporting parallel group randomised trials. Int J Surg. 2012;10:28-55.

12. AAPD. Guideline on Behavior Guidance for the Pediatric Dental Patient. Pediatric dentistry. 2015;37:57.

13. Dikmen B. Icdas II criteria (international caries detection and assessment system). J Istanb Univ Fac Dent. 2015;49:63-72.

14. Dhar V, Marghalani AA, Crystal YO, Kumar A, Ritwik P, Tulunoglu $\mathrm{O}$, et al. Use of vital pulp therapies in primary teeth with deep caries lesions. Pediatric Dentistry. 2017;39:146E-59E.

15. Hariri M, Ramdi H, El Alloussi M, Chhoul H. The Hall Technique: A Non-conventional Method for Managing Carious Primary Molars. Dentistry. 2016;6:21611122.1000385. 
16. Faul F, Erdfelder E, Lang AG, Buchner A. G*Power 3: a flexible statistical power analysis program for the social, behavioral, and biomedical sciences. Behav Res Methods. 2007;39:175-91.

17. Rosenberger WF, Lachin JM. Randomization in clinical trials: theory and practice: John Wiley \& Sons; 2015.

18. Saghaei M. Random allocation software for parallel group randomized trials. BMC Med Res Methodol. 2004;4:26.

19. Doig GS, Simpson F. Randomization and allocation concealment: a practical guide for researchers. J Crit Care. 2005;20:187-91; discussion 91-3.

20. Bang H, Ni L, Davis CE. Assessment of blinding in clinical trials. Control Clin Trials. 2004;25:143-56.

21. Banting DW, Amaechi BT, Bader JD, Blanchard P, Gilbert $\mathrm{GH}$, Gullion CM, et al. Examiner training and reliability in two randomized clinical trials of adult dental caries. J Public Health Dent. 2011;71:335-44.

22. Dean JA. McDonald and Avery's Dentistry for the Child and Adolescent-E-Book: Elsevier Health Sciences; 2015.

23. Full CA, Walker JD, Pinkham JR. Stainless steel crowns for deciduous molars. J Am Dent Assoc. 1974;89:360-4.

24. Innes NP, Ricketts DN, Evans DJ. Preformed metal crowns for decayed primary molar teeth. The Cochrane database of systematic reviews. 2007:CD005512.
25. Subcommittee AAoPDCAC--PT, Affairs AAoPDCoC. Guideline on pulp therapy for primary and young permanent teeth. Pediatric Dentistry. 2005;27(7 Suppl):130.

26. Innes NP, Evans DJ, Stirrups DR. Sealing caries in primary molars: randomized control trial, 5-year results. J Dent Res. 2011;90:1405-10.

27. Innes NP, Stirrups DR, Evans DJ, Hall N, Leggate M. A novel technique using preformed metal crowns for managing carious primary molars in general practice - a retrospective analysis. Br Dent J. 2006;200:451-4; discussion 44.

28. Ebrahimi M, Shirazi AS, Afshari E. Success and Behavior During Atraumatic Restorative Treatment, the Hall Technique, and the Stainless Steel Crown Technique for Primary Molar Teeth. Pediatr Dent. 2020;42:187-92.

29. Binladen H, Al Halabi M, Kowash M, Al Salami A, Khamis AH, Hussein I. A 24-month retrospective study of preformed metal crowns: the Hall technique versus the conventional preparation method. European archives of paediatric dentistry : official journal of the European Academy of Paediatric Dentistry. 2020.

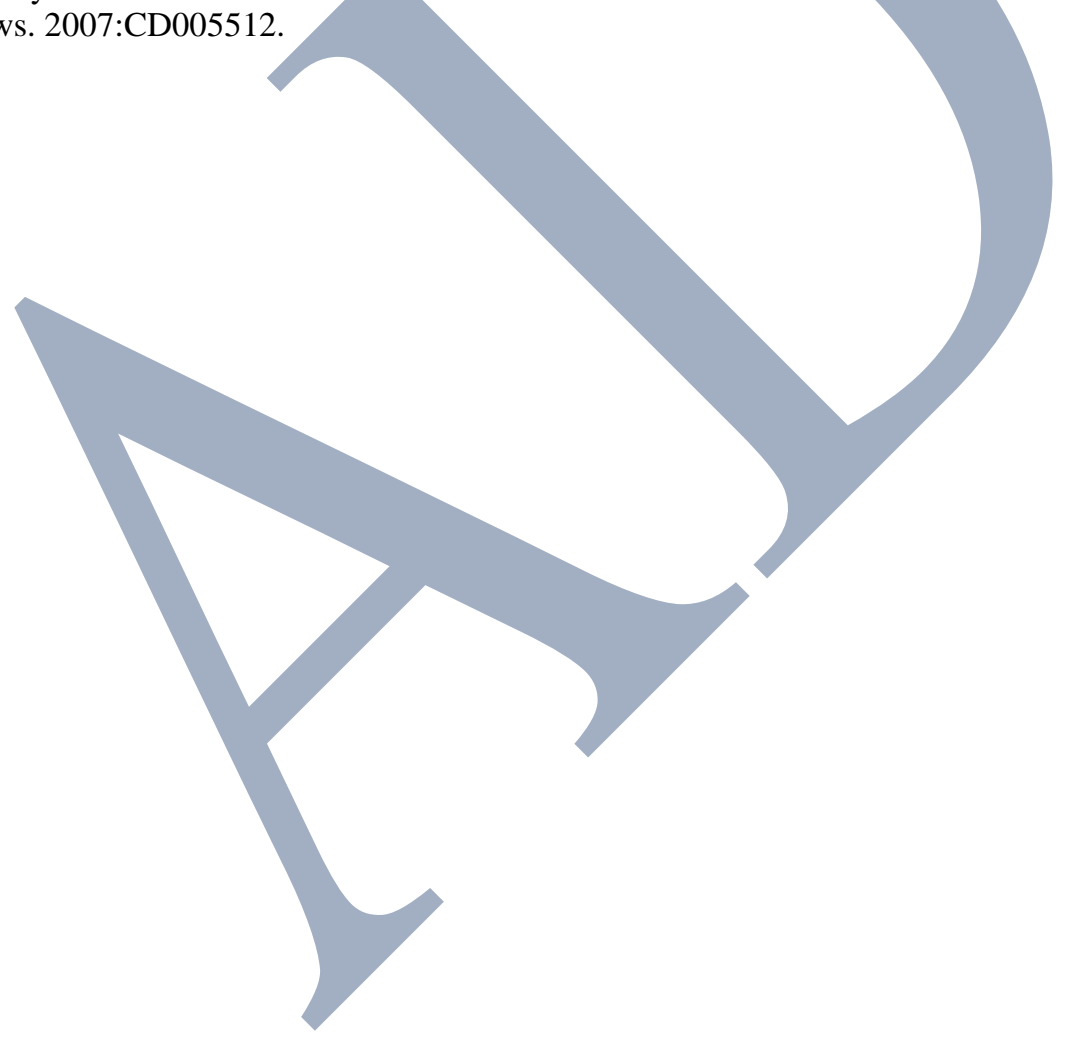

